\title{
The Development of Multi-functional Alternating Current Grid Simulator
}

\author{
ZHANG Yan-Yu
}

\author{
(Hunan Railway Professional Technology College, ZhuZhou Hunan 412001)
}

Keywords: grid simulator; distributed power generation; power system fault; fundamental mode; harmonic mode

\begin{abstract}
In order to adapt to the grid test study a high power alternating current grid simulator that can simulate the network for various status and faults is proposed. The simulator could work in four quadrant operations, so that energy generated by distributed systems could flow into grid. The fundamental voltage and harmonic voltage are controlled respectively: multiple feedback loop control scheme is applied in fundamental mode and effective value feedback control scheme is applied in harmonic mode. The DSP and FPGA platform are used for implementing the system control and the experimental results show that the performance of the simulator is good and has high stability.
\end{abstract}

\section{Introduction}

With more and more distributed generation system connected to the grid, national grid company are the distributed generation system proposed new strict requirements, which requires the grid fault occurs in the distributed generation system can continue and network operation and the issue of active or reactive power to support grid. However to provide standard three-phase sine voltage for the purpose of AC power network voltage drop and unbalanced all grid fault does not see more, therefore, the distributed generation system tested, only through the grid is difficult to achieve these faults, need to can realize electric network fault of the grid simulator researc ${ }^{[1-2]}$.

The literature [3] powered grid voltage sag simulator, is unable to realize energy feedback, not suitable for distributed generation system; The literature [4] established power system simulator, and the DQ decoupling control method to realize zero steady-state error, but the system function is too single, can not achieve the two-way flow of energy; The literature [5] proposed a novel simulation grid control testing equipment, the system based on digital control technology, analog voltage drop, three-phase unbalance and harmonic superposition various grid status and fault, but its use of resonant regulator, makes the control system more complex. The grid simulator or test equipment involved in the above research, or the function of a single can only simulate several grid faults, or control complex.

In view of the above problems, in order to meet the needs of distributed generation grid is proposed in this paper a multifunctional grid simulator, multi function AC grid simulator adopts advanced control strategy can not only were four quadrant operation, to achieve energy bidirectional flow, but also provide the three-phase voltage output and simulation such as voltage sag, voltage unbalance and so on various grid status and fault [6-8]. Calculation of the value of a given voltage under various faults by software programming to achieve grid control strategies of fundamental mode and harmonic mode, fundamental mode adopted based on the instantaneous voltage and current values of the double closed loop control system, harmonic mode the output voltage effective value feedback control, control system has a good effect, high stability, prototype experiment results show that this control scheme is feasible [9]. 


\section{Simulator main circuit topology}

\subsection{Simulator circuit topology}

Three-phase transformer transformer $\mathrm{T}$ and to the frequency conversion power supply, the power input end is equipped with circuit breaker, the front end of the four quadrant rectifier, common DC bus, the input three-phase alternating current rectifier and on the output side by capacitance voltage regulator, to provide a stable DC voltage for the inverter link, the back-end inverter using three single-phase PWM inverter to achieve, each single-phase analog output power of a phase, generated by the rectifier DC voltage inverter are AC voltage, and three single-phase PWM inverter output can be connected to the output transformer T2 of three independent winding, so as to form a three phase with neutral system output. The three-phase PWM rectifier rectifier can make active load generated by the energy back to the grid, can realize bidirectional flow of energy.

The characteristics of the circuit:

1、The input harmonic current is small, the power factor is high, it is advantageous to the test of the grid connected inverter;

2、 Inverter side output using three single-phase PWM inverter structure, each single phase PWM inverter output simulation grid phase, which can achieve a variety of functions required for the grid simulator;

3、The output of the double closed-loop control of output voltage, high steady accuracy, fast response speed, high precision analog current / voltage, overload capacity and high anti disturbance ability。

\subsection{Mathematical model of single phase full bridge inverter}

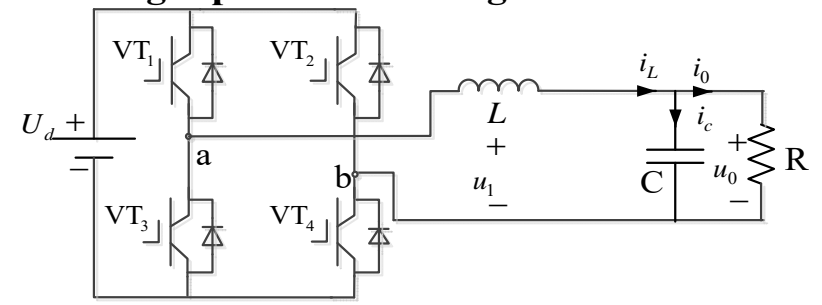

Fig. 1 the single-phase bridge inverter

Fig. 1 is a single phase full bridge inverter circuit, the inductor current and output voltage are regarded as the state variable of the inverter, and the state expression of the single-phase inverter can be:

$$
\begin{aligned}
{\left[\begin{array}{c}
u_{0} \\
i_{L}
\end{array}\right] } & =\left[\begin{array}{cc}
0 & \frac{1}{C} \\
-\frac{1}{L} & -\frac{r}{L}
\end{array}\right]\left[\begin{array}{l}
u_{0} \\
i_{L}
\end{array}\right]+\left[\begin{array}{c}
0 \\
\frac{1}{L}
\end{array}\right] u_{1}+\left[\begin{array}{c}
-\frac{1}{C} \\
0
\end{array}\right] i_{0} \\
y & =\left[\begin{array}{ll}
1 & 0
\end{array}\right]\left[\begin{array}{c}
u_{0} \\
i_{L}
\end{array}\right]
\end{aligned}
$$

From equation (2) and (3) we can see that the single-phase inverter control system for the two order system, a double input single output.

\section{Simulator control strategy}

\subsection{Fundamental and harmonic mode control strategy}

And corresponding to the main circuit control part includes the rectifier and inverter of fundamental and harmonic modes control strategy links two parts of control, rectifier using PWM control to realize four quadrant operation to enable distributed generation system can feed energy back into the grid.

The output of the power system simulator is divided into the fundamental mode and the 
harmonic mode. The fundamental mode is based on the double closed loop control mode, which is based on the instantaneous value of voltage and current, which can not meet the requirements of high performance index, but also can improve the response speed and enhance the stability of the system. In the current loop, negative feedback current is introduced to speed up the dynamic response of the inverter, enhance the ability to adapt to the nonlinear load disturbance, and reduce the harmonic content of the output voltage. Fig. 2 for the fundamental mode of the system under the control box, Fig. 3 for the high order harmonic mode under the double closed-loop control block diagram.

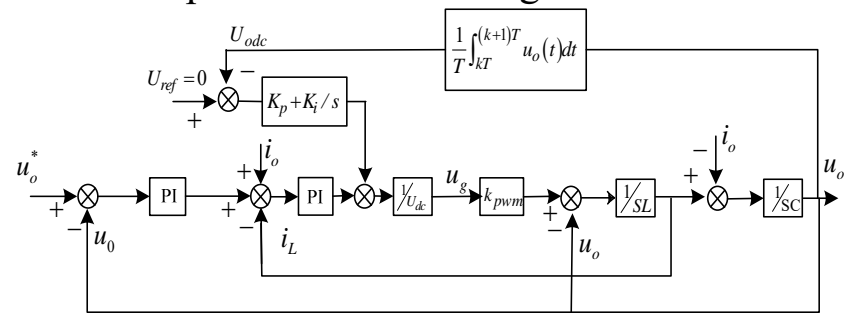

\section{Fundamental mode}

Fig.2 double closed-loop control block diagramunder

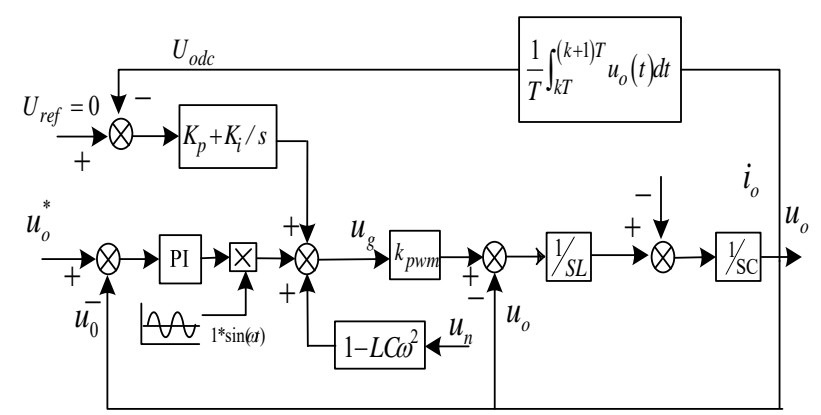

Fig. 3 double closed-loop control block diagram under higher harmonic mode

In the fundamental mode, the LC filter will have a certain effect on the high order harmonics in the output voltage. If the LC filter in Figure 2 is ideal, the transfer function of the filter is:

$$
G_{L C}(s)=\frac{1}{L C S^{2}+1}
$$

In the formula, $\mathrm{L}, \mathrm{C}$, respectively, the filter inductor and filter capacitor 。

The largest known corrugated inductor current determines the output filter inductance under PWM modulation L. Its value is usually equal to about corrugated sinusoidal output current peak of $10 \%$, i.e.

$$
\Delta I_{\max }=10 \% \times 2 \times \frac{S}{U_{m}}
$$

In the formula, $\mathrm{S}$ is the output power rating, $\mathrm{Um}$ is the output voltage amplitude $1 / 5$.

According to the research results of [6], available

$$
L \geq \frac{U_{d}}{8 \Delta I_{\max } f_{s}}
$$

In the formula, Ud is the DC bus voltage, and FS is the switching frequency.

Capacitor values are generally determined by the cutoff frequency of the filter. Because this paper adopts SPWM modulation technology, the harmonic frequency is 2 times of the switching frequency, and the cut-off frequency is about $10 / 1$ :

$$
\frac{1}{2 \pi \sqrt{L C}} \leq \frac{2 f_{s}}{10}
$$

Therefore, the value of the capacitor can be got.

$$
C \geq \frac{25}{4 \pi^{2} L f_{s}^{2}}
$$




\subsection{Analysis of voltage and current double loop control strategy}

Through the research show that the voltage outer loop current inner loop proportional integral (Pipi) control strategy than the outer voltage loop current loop (PIP) ratio control strategy in some aspects of performance are slightly higher, but in terms of stability, (Pipi) control strategy because of the inner current loop integral constant is too large, the use of digital control, leading to the processor's data overflow, resulting in the instability of the control system. Therefore, this paper will use the voltage and current loop outer loop proportional PIP control strategy, its block diagram as shown in Fig. 4.

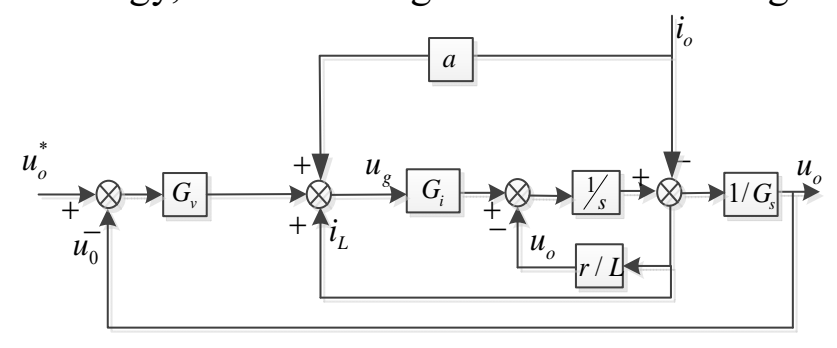

Fig. 4 The block diagram of Double loop

In Fig. $4 \mathrm{G}_{\mathrm{v}}=k_{\mathrm{p}}+k_{\mathrm{i}} / \mathrm{s}, \mathrm{G}_{\mathrm{i}}=k$, The closed loop and open loop transfer function of the inductor current inner loop are respectively

$$
\begin{gathered}
G_{i c l}=\frac{C k s}{L C s^{2}+(C r+C k) s+1} \\
G_{\text {iop }}=\frac{C k s}{L C s^{2}+C r s+1}
\end{gathered}
$$

According to the formula (8) and (9), the voltage outer loop and the closed-loop transfer function are respectively

$$
G_{\text {uop }}=\frac{k\left(k_{p} s+k_{i}\right)}{L C s^{3}+(C r+C k) s^{2}+s}
$$

The closed-loop transfer function of the voltage outer loop and the output impedance of the system are respectively:

$$
G_{u c l}=\frac{k_{p} k s+k_{i} k}{L C s^{3}+C(r+k) s^{2}+\left(1+k_{p} k\right) s+k_{i} k}
$$

The output impedance of the system is

$$
G_{i o}=\frac{k S^{3}+(r+k-a k) s}{L C s^{3}+C(r+k) s^{2}+\left(r+k_{p} k\right) s+k_{i} k}
$$
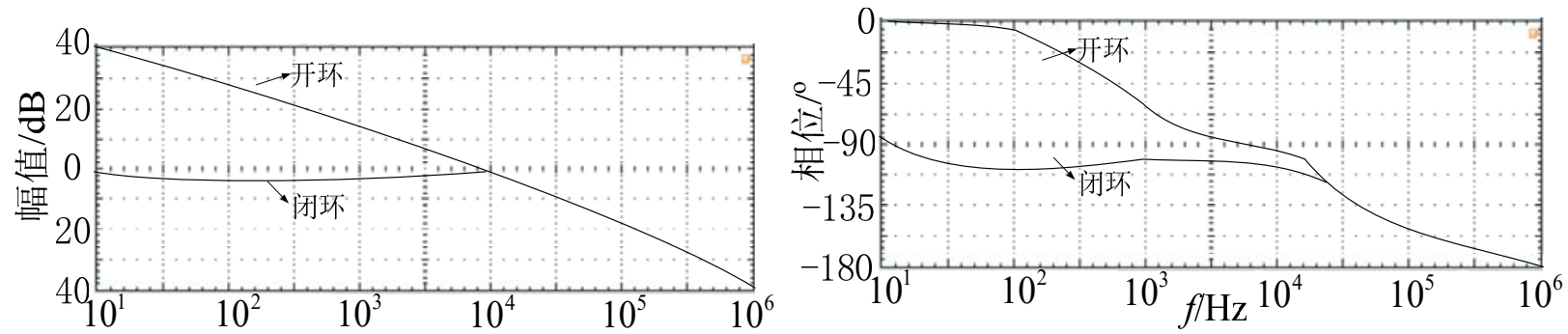

Fig.5 Voltage outer loop and closed loop frequency characteristic

According to the pole method, the parameters of PIP are obtained: $k_{\mathrm{p}}=0.0112, k_{\mathrm{i}}=23.7832$, $\mathrm{k}=199.2$.

From Fig. 5 for voltage outer loop the open-loop frequency response characteristics can be seen voltage outer

loop, the voltage regulator makes the phase margin of about 70 degrees, amplitude margin is large enough to ensure the stability; from Fig. 5 for voltage outer ring loop frequency characteristics can be seen control system fundamental closed-loop gain is close to 1, the phase with the smaller the high stability. 


\section{Conclusion}

This paper introduces a multifunctional grid simulator, the simulation of AC three-phase output voltage can be adjusted independently, can simulate the three-phase unbalance, voltage sag faults, introduces a multifunctional grid simulator, the output of the simulator is divided into fundamental mode and harmonic mode, using the base wave mode voltage and current double closed-loop control scheme the instantaneous value based on the harmonic mode of the output voltage effective value feedback control, a given voltage because of the programmability of only need to meet the different requirements of output voltage by simple programming can, practical simulator control system is very strong with DSP and FPGA, the experimental results show that the simulator has good performance, high stability, for power system simulator more power, this conclusion is also suitable, can be used as reference for analysis and design.

\section{Reference}

[1] Zhang R, Cardinal M. A Grid Simulator with Control of Single-phase Power Converters in $d, q$ Rotating Frame [C]. Power Electronics Specialists Conference. 2002: 1431-1436.

[2] Poh Chiang Lohan, Newman M J. A Comparative Analysis of Multiloop Voltage Regulation Strategies for Single and Three-phase UPS Systems [J]. IEEE Trans. on Power Electronics, 2003, 18(5): 1176-1185.

[3] E R Collins. A Three-phase Sag Generator for Testing Industrial Equipment [J]. IEEE Trans. on Power Delivery. 1996, 11(1): 526-532.

[4] Zhang R , Cardinal M , Szczesny P. A Grid Simulator withControl of Single-phase Power Converters in d, q Rotating Frame[A]. Power Electronics Specialists Conference[C]. 2002 : 1431-1436.

[5] Sebastian A Richter, Jochen von Bloh. Control of a Medium-voltage Test Generator[A]. IEEE Power Electronics Specialists Conference [C]. 2008 : 3787-3793.

[6] J.Carter,C.J.Goodman." Analysis Of The Singal-phase Four-quadrant PWM Converter resulting in steady state and small-singal dynamic models". IEEE Proc Electr. 1997, 144(4):241-247. 